\title{
High efficacy of two artemisinin-based combinations: artesunate + sulfadoxine-pyrimethamine and artemether-lumefantrine for falciparum malaria in Yemen
}

\author{
Ahmed A. Adeel ${ }^{1 *}$, Niaz Abdo Saeed ${ }^{2}$, Adel Aljasari², Amar M. Almohager ${ }^{3}$, Mohamed H. Galab³, \\ Amar AlMahdi ${ }^{3}$, Mansor H. Mahammed ${ }^{3}$, Mohammed AlDarsi ${ }^{3}$, Yahiya A. Salaeah ${ }^{3}$, Hoda Atta ${ }^{4}$, \\ Ghasem Zamani ${ }^{4}$, Marian Warsame ${ }^{5}$, Amy Barrette ${ }^{5}$, Hanan El Mohammady ${ }^{6}$ and Rania A. Nada ${ }^{6}$
}

\begin{abstract}
Background: Artesunate + sulfadoxine-pyrimethamine (AS + SP) has been the first-line treatment and artemetherlumefantrine (AL) the second-line treatment for uncomplicated falciparum malaria in Yemen since 2005. This paper reports the results of studies conducted to monitor therapeutic efficacy of these two drugs in sentinel sites in Yemen.

Methods: Eight therapeutic efficacy studies were conducted in six sentinel sites during the period 2009-2013 in Yemen. Five studies were for the evaluation of AS + SP (total of 465 patients) and three studies (total of 268 patients) for the evaluation of AL. The studies were done according to standard WHO protocol 2009 with 28-day follow-up.

Results: In the evaluation of AS + SP, the PCR-corrected cure rate was $98 \%$ (95\% Cl 92.2-99.5\%) in one site and $100 \%$ in all of the other four sites. In the sites where AL was evaluated, the PCR-corrected cure rate was $100 \%$ in all the sites. All patients were negative for asexual parasitaemia on day 3 in both the AS + SP and the AL groups. There was a higher rate of clearance of gametocytaemia in the AL-treated group when compared with the AS + SP groups from day 7 onwards.

Conclusion: AS + SP remains the effective drug for uncomplicated falciparum malaria in Yemen. AL is also highly effective and can be an appropriate alternative to AS + SP for the treatment of falciparum malaria. AL demonstrated a higher efficacy in clearing microscopic gametocytaemia than AS + SP.

Trial registration: Trial registration number ACTRN12610000696099
\end{abstract}

Keywords: Yemen, Artesunate, Sulfadoxine-pyrimethamine, Anti-malarial drugs, Drug resistance, Plasmodium falciparum, Gametocytaemia, Artemether-lumefantrine

\section{Background}

There are four major epidemiological strata of malaria in the Republic of Yemen. These are coastal plain, foothills, mountains, and the island of Socotra. Malaria-free

\footnotetext{
*Correspondence: aaadeel@yahoo.com

${ }^{1}$ College of Medicine, King Saud University, Riyadh, Saudi Arabia Full list of author information is available at the end of the article
}

zones include the mountain plateau and arid slopes from the highlands to the desert. In 2013, $25 \%(6,100,000)$ of a total population of $24,400,000$ lived in areas of high transmission (>1 case/1000), $53 \%(12,900,000)$ in areas of low transmission $(0-1$ case $/ 1000)$ and $22 \%(5,400,000)$ in malaria-free areas [1]. Most of the cases (99\%) are due to Plasmodium falciparum and only $1 \%$ due to Plasmodium vivax. No indigenous malaria has been detected in

\section{Biomed Central}

(c) 2015 Adeel et al. This article is distributed under the terms of the Creative Commons Attribution 4.0 International License (http://creativecommons.org/licenses/by/4.0/), which permits unrestricted use, distribution, and reproduction in any medium, provided you give appropriate credit to the original author(s) and the source, provide a link to the Creative Commons license, and indicate if changes were made. The Creative Commons Public Domain Dedication waiver (http://creativecommons.org/ publicdomain/zero/1.0/) applies to the data made available in this article, unless otherwise stated. 
Socotra since 2006 [2]. Malaria transmission in Yemen differs between the regions. In the coastal areas, peak transmission occurs in winter (October-April), while in the mountainous hinterland areas it usually peaks in summer (May-September). However, in highland areas located above $2000 \mathrm{~m}$ above sea level, transmission occurs throughout the year [3]. The geographic location of Yemen makes it highly relevant to the malaria elimination efforts of its northern neighbour (Saudi Arabia) and for the prevention of re-introduction of malaria of its eastern neighbour (Oman). Given that Yemen contributes over $98 \%$ of the malaria burden of the Arab Peninsula, the achievement of the targets of 'malaria Free Arabian Peninsula Initiative' depends heavily on the disease situation in Yemen [2].

Most of the early studies on anti-malarial drug efficacy that were carried out in Yemen in the 1980s and the 1990s were done in the southern parts of the country. These were mainly in vivo studies based on the standard WHO seven-day test to assess response of falciparum malaria to chloroquine (CQ). The studies, which were conducted by WHO consultants for malaria control, reported no significant levels of $\mathrm{CQ}$ resistance at the time [4]. In 2002, the National Malaria Control Programme (NMCP) established sentinel sites for monitoring the therapeutic efficacy of anti-malarial drugs to $P$. falciparum based on the earlier versions of standard WHO protocol [5]. Since then, 17 studies have been conducted by the NMCP, covering $C Q$, sulfadoxine-pyrimethamine (SP) and amodiaquine (AQ) monotherapy and more recently, the artemisinin-based combinations of artesunate + amodiaquine $(\mathrm{AS}+\mathrm{AQ})$, artesunate + sulfadoxine-pyrimethamine (AS + SP) and artemether + lumefantrine (AL) (Adel Aljasary, pers. comm.). The first therapeutic tests on CQ were conducted in 2002-03 [6,7] based on the 2001 WHO protocol [5]. By 2004, these tests revealed high rates of treatment failures with $C Q$ and the results were summarized in WHO's report on global monitoring of susceptibility of Plasmodium falciparum to antimalarial drugs, which cited nine therapeutic efficacy studies in Yemen with median treatment failure rate of $42.4 \%$ (range 9-57 \%) for CQ and one trial for SP with no treatment failure [8].

The emergence and spread of drug-resistant malaria has been a major factor in the global resurgence of falciparum malaria in the 20th Century. Drug resistance has been implicated in the spread of malaria to new areas and re-emergence of malaria in areas where the disease had been eliminated. Drug resistance has also played a significant role in the occurrence and severity of epidemics in some parts of the world [9]. In Yemen, the spread of CQ-resistant falciparum malaria was associated with deterioration of the malaria situation in terms of disease prevalence and clinical impact [10]. The experience of Yemen with CQ resistance brought attention to the significance of surveillance of the efficacy of anti-malarial drugs as a main activity of the NMCP.

In 2005, Yemen switched to artemisinin-based combination therapy (ACT) for the treatment of uncomplicated falciparum malaria as per the recommendation of WHO [11]. The selection of the exact ACT is based on consideration of the options and the local resistance patterns for the partner drug [12, 13]. Efficacy trials done by the NMCP in Ibb in 2004 found that treatment failure rate after AQ monotherapy was $44 \%$ and with AS + AQ $18.5 \%$ (Adel Aljasary, pers. comm.). AS + AQ was excluded as an option in Yemen and led to selection of AS + SP as the first-line drug and AL as the secondline drug for uncomplicated falciparum malaria (Adel Aljasary, pers. comm.).

AS + SP has been evaluated extensively in adults and children with uncomplicated malaria in other parts of the world and was found to be sufficiently efficacious in areas where 28-day cure rates with SP alone exceeded $80 \%[13,14]$. AL is currently one of the most widely used ACT for treatment of uncomplicated falciparum malaria. The previous four-dose regime was associated with $15 \%$ treatment failure, but the now-recommended, six-dose regime has shown higher efficacy [15]. This paper presents the results of studies conducted to assess therapeutic efficacy of AS + SP and AL against falciparum malaria in Yemen from 2009 to 2013. In addition, the impact of these two ACTs on microscopic gametocytaemia were evaluated in the study population. These studies were carried in the framework of monitoring therapeutic efficacy of anti-malarial drugs.

\section{Methods}

\section{Study design and patients}

The studies were conducted in six sentinel sites representing the different malaria-endemic provinces of the country (see Fig. 1). Each study was timed to coincide with the peak of the malaria transmission in the area. The design of the study was an open-label, one-arm, prospective evaluation of clinical and parasitological responses to directly observed treatment for uncomplicated malaria. The WHO protocol for assessing therapeutic efficacy [16] was used. Cases were enrolled in the study, after obtaining the patients' or parents'/guardians' written consent, if they were aged 6 months and above, had symptoms compatible with uncomplicated clinical malaria with fever (body temperature $\geq 37.5^{\circ} \mathrm{C}$ ) or history of fever in the previous $24 \mathrm{~h}$ and mono-infection of $P$. falciparum with parasite density of 500-200,000 asexual parasites/ 


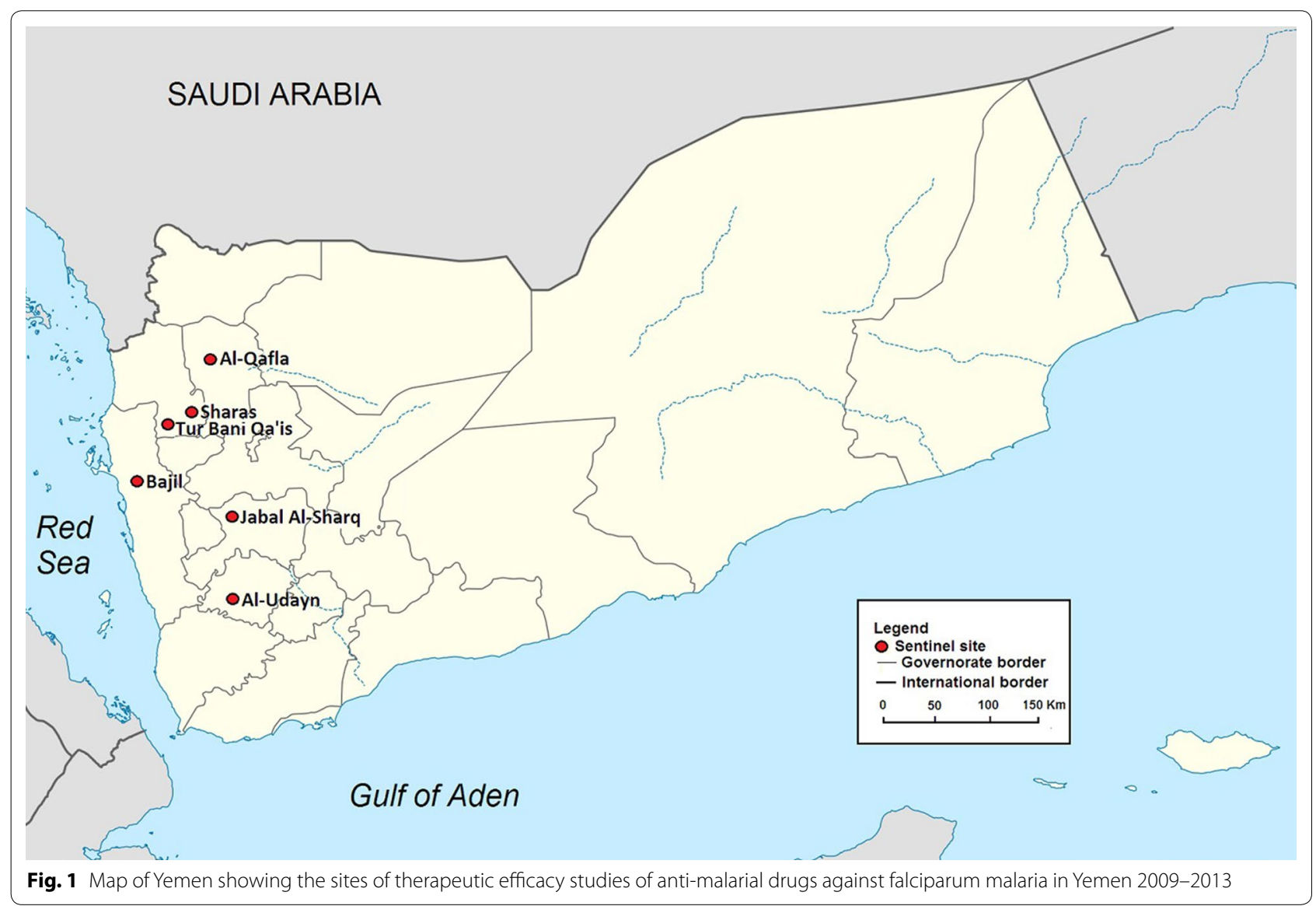

$\mu$ l. A patient was excluded if she/he had a febrile illness other than malaria or severe and complicated falciparum malaria.

Pregnant women and females of reproductive age who could not be tested for pregnancy were excluded. Pregnancy testing of unmarried women and female minors aged 12-17 years in Yemen is not acceptable according to the local customs and culture.

\section{Treatment and laboratory analysis}

The methods of treatment, follow-up and analysis of outcomes was based on WHO guidelines [16]. After obtaining a written informed consent, a complete medical history was obtained and a complete physical examination was performed. Blood was collected for microscopy and on filter paper on day 0 before treatment and on days $2,3,7,14,21,28$ or on any other day if the patient returned spontaneously.

\section{Treatment regimen}

All patients received a standard regimen of the drug being tested [12]. In the AS + SP studies the patients received a dose of $4 \mathrm{mg} / \mathrm{kg} /$ day artesunate once a day for 3 days and a single administration of $25 / 1.25 \mathrm{mg} / \mathrm{kg} \mathrm{SP}$ on day 0 , with a therapeutic dose range between 2 and $10 \mathrm{mg} / \mathrm{kg} /$ day artesunate and 25-70/1.25-3.5 mg/kg SP. In studies on $\mathrm{AL}$, the patients were given $\mathrm{AL}$ (Coartem ${ }^{\circledR}$, Novartis Pharmaceutical Corporation) according to body weight bands. Patients weighing 5-14 kg received one tablet (20 mg artemether plus $120 \mathrm{mg}$ lumefantrine) per dose, those weighing $15-24 \mathrm{~kg}$ received two, those weighing 25-34 kg three tablets, and those weighing $\geq 35 \mathrm{~kg}$ received four tablets. In total, six doses were administered at hours $0,8,24,36,48$, and 60 . Study medications were provided by WHO. The intake of all doses of treatment was directly observed. All drugs were within their expiry period, and batch number and expiry date were recorded in each case record form (CRF). If a patient vomited within $30 \mathrm{~min}$ of treatment, a full dose was readministered. All patients were followed up on days 1 , $2,3,7,14,21$, and 28 . On each of these follow-up visits clinical and parasitological assessments were repeated. Patients were also asked to report to the clinic at any time if new or recurrent symptoms occurred. 


\section{Rescue medication}

All patients who failed to be cured with AS + SP were treated with AL. Those who failed treatment with AL were treated with quinine orally at $10 \mathrm{mg}$ salt $/ \mathrm{kg}$ three times daily for 7 days.

\section{Malaria microscopy}

Thick and thin blood films for parasite counts were obtained from each patient and examined at screening and on days $2,3,7,14,21,28$ or on any other day if the patient returned spontaneously and parasitological reassessment was required. Blood smears were stained with $2.5 \%$ Giemsa for $45 \mathrm{~min}$ and examined at a magnification of $1000 \times$ to identify the parasite species and to determine the parasite density.

The thick blood smear was used to calculate the parasite density, by counting the number of asexual parasites against 200 white blood cells (WBC) with a hand tally counter. The count was terminated when either 500 parasites or $200 \mathrm{WBC}$ were reached, whichever came first. A blood smear was declared negative after examination of 1000 WBC revealed no asexual parasites. Parasite density, expressed as the number of asexual parasites per $\mu \mathrm{l}$ of blood, was calculated by dividing the number of asexual parasites by the number of WBC counted and then multiplying by an assumed WBC density of 8000 per $\mu l$.

For quality assurance, all blood smears were re-read by a second microscopist. Blood smears with non-concordant results (differences in species or differences in parasite density of $>50 \%$ ) were re-read by a third microscopist and the average parasite density of the two most concordant counts was used.

\section{Genotyping of malaria parasites}

Two to three drops of blood were collected through finger pricks on filter paper (Whatmann No 3) during enrolment and each time blood smears were required, according to the protocol, on and after day 7. Specimens were labelled anonymously (Patient ID number, day of follow-up, date), kept in individual plastic bags with desiccant pouches and protected from light, humidity and extreme temperature until analysed. The specimens were genotyped to distinguish between recrudescence and new infections, according to methods recommended by WHO [17]. Blood spots were tested using nested polymerase chain reaction (PCR) targeting polymorphic variant genes $m s p 1, m s p 2$ (merozoite surface proteins) and glurp (glutamate-rich protein). Subsequently, gel analysis was performed using Bionumerics V.5.10 in order to determine the size of the amplified gene targets. Identification of recrudescence and new infections was performed according to WHO guidelines [17].

\section{Classification of treatment outcome}

Treatment outcomes were classified on the basis of an assessment of the parasitological and clinical outcome of anti-malarial treatment according to WHO guidelines [16]. A patient was classified as having either early treatment failure (ETF), late clinical failure (LCF), late parasitological failure (LPF), or an adequate clinical and parasitological response (ACPR), as defined in guidelines [16]. Patients who were lost to follow-up, had re-infections or unknown PCR were excluded from the per-protocol analysis of treatment outcomes, but included in the Kaplan-Meier analysis until the day of withdrawal from the study.

\section{Drug tolerability and safety}

Patients were assessed clinically for drug tolerability. Both adverse events and serious adverse events were monitored at enrolment and on each of the follow-up visit. An adverse event was defined as any untoward medical occurrence irrespective of its suspected relationship to the study medications. Serious adverse event included untoward medical occurrence requiring hospitalization or resulting in death.

\section{Sample size and statistical analysis}

The treatment failure rate of the two drugs in the study areas was estimated to be $5 \%$. At a confidence level of $95 \%$ and a precision estimate of $5 \%$, a minimum of 73 patients had been planned to be enrolled in each site. With a $20 \%$ increase to allow loss to follow-up and withdrawals during the 28-day follow-up period, 87 patients were targeted to be included in the study per site per drug. Data were double entered and validated using a programme developed by WHO [16]. Per protocol and Kaplan-Meier survival analysis were used to evaluate the treatment outcome and only patients who could be evaluated on the respective days of follow-up were included in the analysis. The proportion of positive blood films on day 3 was recorded. Geometrical means of parasite density on day 0 were calculated for each site.

Statistical analysis was performed using SPSS $22^{\circledR}$ to generate descriptive statistics and analyse data (SPSS Inc. Chicago, USA). A $2 \times 2$ Chi square table was used to analyse associations between proportions using Epi-Info 7 (Centers for Disease Control and Prevention, Atlanta, GA, USA and WHO, Geneva, Switzerland).

\section{Ethical considerations}

Permission to conduct the studies was obtained from the General Doctorate for Research and Studies, Ministry of Health, Yemen, which is the national body with oversight to ethically review research proposals involving human subjects, and WHO Ethical Research Committee. 
Local authorities (community leaders) of the study areas were informed of the study objectives, procedures and duration and their permission was sought. Individual informed consent was obtained from adults and parents/ guardians of children. Those who were illiterate selected a witness not related to the research team to sign on their behalf.

\section{Results}

Eight therapeutic efficacy studies were conducted in six sentinel sites in Yemen to assess the therapeutic efficacy of AS + SP (five studies) and AL (three studies) during the period November 2009 to March 2013. Table 1 shows the demographic and clinical characteristics in the study population in each site. The age of patients ranged between 6 months and 75 years. The percentage of children under 5 years of age varied by study, ranging between $8.6 \%$ in Tur Bani Qa' is 2013 to $56 \%$ in al-Udayn 2010. There were also differences in gender composition in the different studies, the percentage of males ranged between 46.7 and $72 \%$. The mean temperature at baseline showed variation ranging between 37.6 and $38.0{ }^{\circ} \mathrm{C}$ and the geometric mean asexual parasitaemia/ $\mu$ l varied from 1729 to $14,543 / \mu \mathrm{l}$.

Table 2 shows details of the outcomes of all the studies. In the AS + SP studies, a total of 465 patients with falciparum malaria who satisfied the inclusion criteria were enrolled. The study was completed by 432 patients, with 426 cases classified on day 28 as ACPR. There were three LCF and two LPF. In addition, there were ten patients lost to follow-up and 24 withdrawals. The reasons for withdawal were quality control detecting that parasite count at baseline was too low (18 cases), mixed infection (two cases), underdose (one case), and withdrawal of consent by patient (three cases). According to the follow-up and analysis plan, cases initially classified as LCF or LPF were checked by PCR to differentiate recrudescence from re-infection. PCR-correction confirmed recrudescence in two of the LCF cases.

In the individual studies and after PCR-correction Kaplan-Meier analysis, the cure rate with AS + SP ranged between 98 and $100 \%$ in the different sites. The two cases of treatment failure were in Bajil 2013. The first case (LCF) was a 2 year old male who presented on day 28 with a history of fever, his blood film showed $880 P$. falciparum asexual stages/ $\mu \mathrm{l}$. The second case (LCF) was a 5 year old female who presented on day 28 with a recent history of fever and her blood film was positive with 45,454 P. falciparum asexual stages/ $\mu$ l. Both cases had treatment doses within the therapeutic range.

In the AL studies, a total of 268 patients with falciparum malaria satisfied the inclusion criteria (Table 1 ). The studies were completed by 252 patients with 245 cases classified on day 28 as ACPR, two cases as LPF in JabalAl-Sharq 2010, and five cases in Tor-Bani-Qa'is as LCF. In addition, there were six patients lost to follow-up and ten withdrawals. The reasons for withdrawal was quality control detecting low baseline parasitaemia (seven cases) and withdrawal of consent (three cases). According to the follow-up and analysis plan, cases initially classified as LCF or LPF were checked by PCR for recrudescence or re-infection, which found the five cases initially classified at LCF in Tur-Bani-Qa'is to be re-infections; the two cases initially classified as LPF (from Jabal-Al-sharq) were excluded from the PCR-corrected analysis because they had unknown PCR. In the PCR-corrected analysis and after Kaplan-Meier analysis, the cure rate following AL was $100 \%$ in all the three sites tested. All cases except one had cleared asexual parasitaemia by day 3 . Details of the PCR-uncorrected treatment failure cases were as follows: the first one was a 4 year-old female, who presented with history of fever and with 1400 asexual stages/ $\mu$ l on day 0 . Low parasite count persisted on day 2 (520 asexual stages/ $\mu \mathrm{l})$, day 3 (320 asexual stages/ $\mu \mathrm{l})$ and day 7 , when

Table 1 Patient characteristics on admission, Yemen (2010-2013)

\begin{tabular}{|c|c|c|c|c|c|c|c|c|c|c|}
\hline \multirow[t]{2}{*}{ Drug } & \multirow[t]{2}{*}{ Site } & \multirow[t]{2}{*}{ Year } & \multirow[t]{2}{*}{$\mathrm{n}$} & \multirow[t]{2}{*}{ Males } & \multirow[t]{2}{*}{$(\%)$} & \multicolumn{2}{|c|}{ Age $<5$ years } & \multirow{2}{*}{$\begin{array}{l}\operatorname{Temp}^{\circ} \mathrm{C} \\
\text { Mean (SD) }\end{array}$} & \multirow{2}{*}{$\begin{array}{l}\text { Febrile patients }{ }^{\mathrm{a}} \geq 37.5 \\
\mathrm{n}(\%)\end{array}$} & \multirow{2}{*}{$\begin{array}{l}\text { Parasitaemia }(/ \mu \mathrm{L}) \\
\text { Geometric mean }(95 \% \mathrm{Cl})\end{array}$} \\
\hline & & & & & & n (\%) & $95 \% \mathrm{Cl}$ & & & \\
\hline $\mathrm{AS}+\mathrm{SP}$ & Al-Udayn & 2010 & 85 & 44 & $(51.8)$ & $48(56.5)$ & $(45.3-67.2)$ & $37.9(0.2)$ & $70(82.4)$ & 3429 (2643-4449) \\
\hline$A S+S P$ & Sharas & 2010 & 93 & 52 & $(55.9)$ & $31(33.3)$ & $(23.9-43.9)$ & $37.7(0.1)$ & $56(60.2)$ & 9088 (6947-11,890) \\
\hline$A S+S P$ & Tur Bani Qa'is & 2010 & 95 & 58 & $(61.1)$ & $38(40)$ & $(30.1-50.6)$ & $37.9(0.3)$ & $75(78.9)$ & $14543(10,325-18,628)$ \\
\hline$A S+S P$ & Al Qaflaha & 2011 & 90 & 42 & $(46.7)$ & $33(36.7)$ & $(26.7-47.5)$ & $37.6(0.7)$ & $55(61.1)$ & $3238(2506-4184)$ \\
\hline$A S+S P$ & Bajil & 2013 & 102 & 51 & $(50.0)$ & $10(9.8)$ & $(4.8-17.3)$ & $38.0(0.4)$ & $45(44.1)$ & 7011 (5461-8999) \\
\hline $\mathrm{AL}$ & Bajil & $2009-10$ & 80 & 43 & $(53.8)$ & $7(8.8)$ & $(3.6-17.2$ & $37.6(0.4)$ & $74(92.5)$ & $12,606(8707-18,248)$ \\
\hline$A L$ & Jabal Al Sharq & 2010 & 95 & 57 & $(60.0)$ & $14(14.7)$ & $(8.3-23.5)$ & $37.6(0.3)$ & $57(60)$ & 1729 (1399-2137) \\
\hline$A L$ & Tur Bani Qa'is & 2013 & 93 & 67 & $(72.0)$ & $8(8.6)$ & $(3.8-16.2)$ & $38.0(0.1)$ & $81(87.1)$ & $11,872(8698-16,203)$ \\
\hline
\end{tabular}

a Febrile patients: axillary temp $\geq 37.5^{\circ} \mathrm{C}$ 


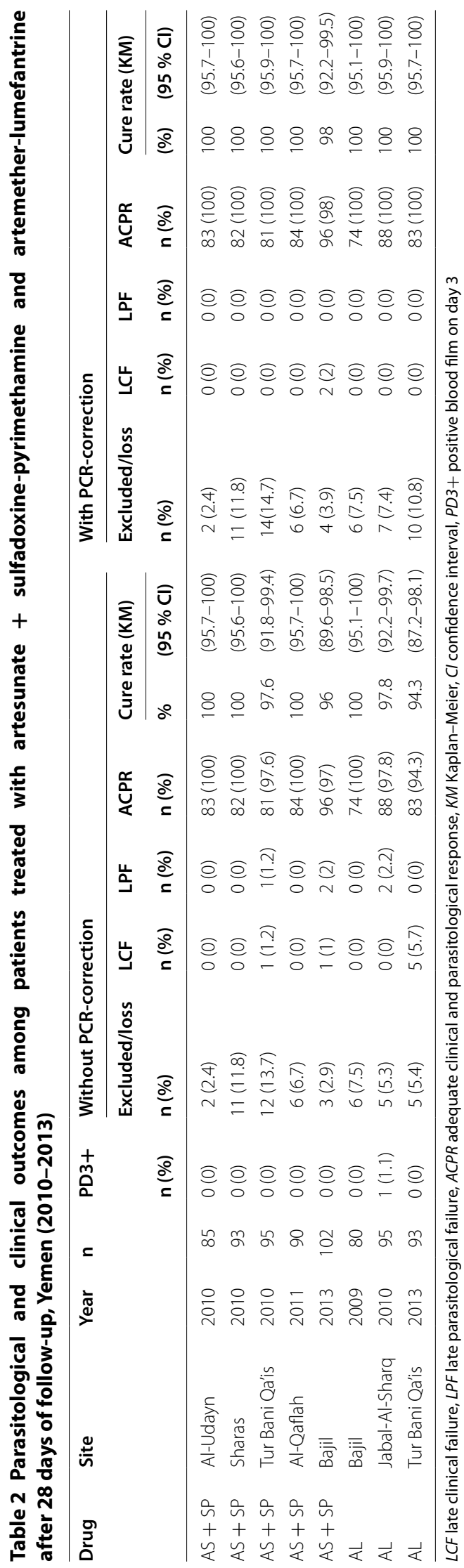


the parasite count went up to 5502 asexual stages/ $\mu$ l. She was classified as LPF, and because her PCR was negative on day 7 she was excluded in the PCR-corrected classification. This was the only case that had microscopic parasitaemia on day 3 . The second case was a 7 year old, who presented with history of fever, parasite density on day 0 was 1200 asexual stages/ $\mu \mathrm{l}$, and had adequate resolution of fever and microscopic parasitaemia but on day 21 the parasite count was 7000 asexual stage/ $\mu$ l. The patient was classified as LPF but excluded from the PCR classification because the PCR was not known. The five cases of LCF in Tur-Bani-Qa'is were all children between 6 and 10 years old, who presented with fever and positive blood film on day 21 (one case) and day 28 (four cases). PCR analysis classified all five cases as re-infections. All therapeutic regimens of the two study drugs were well tolerated and safe.

Figure 2 is a Kaplan-Meier plot (survival analysis) showing gametocyte clearance times in patients who were gametocytaemic at enrolment, after treatment with AL $(n=42)$ and with AS + SP group $(n=91)$. By day 7 , after treatment the proportion with gametocytaemia was significantly lower in the AL group compared with the ASSP group [20/42 (47.6 \%) versus 68/91 (74.7\%), Chi $=9.432, \mathrm{P}=0.002]$. This higher gametocyte clearance rate was observed at the follow-up visits on days 14, 21 and 28. Among the patients who were negative for gametocytes at baseline, more patients in the AS + SP group developed gametocytaemia after treatment than in the AL group, 22/342 (6.4\%) versus 5/214 (2.3\%), respectively, $\mathrm{P}=0.041$, Fisher's exact test.

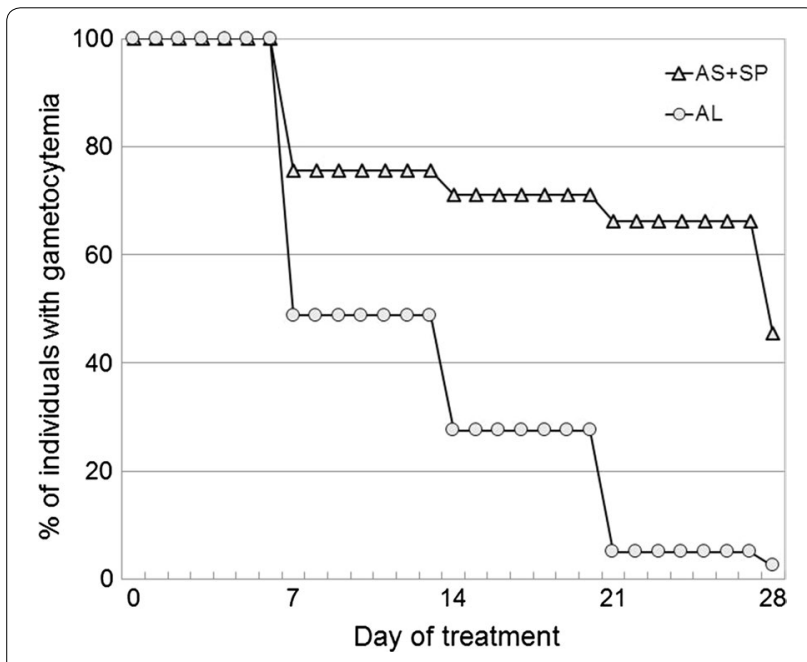

Fig. 2 Time to disappearance of microscopic gametocytaemia in gametocyte-positive individuals at enrolment and following treatment. $A L$ artemether-lumefantrine $(n=42), A S+S P$ sulfadoxinepyrimethamine $(n=91)$

\section{Discussion}

The 28-day parasitological cure observed after first-line AS + SP therapy in the present study exceeded $95 \%$ in all the sites after PCR-correction and also in the uncorrected analyses, meeting the WHO recommendation that cure rates for falciparum malaria should be at least $90 \%$ and preferably $>95 \%$. Detection of asexual parasites on day 3 is an early warning sign of slow clearance of parasites by artemisinin $[18,19]$. In the present study, the complete clearance of asexual parasitaemia in all cases on day 3 after treatment with AS + SP was an indication that the artesunate component is still effective. Since the blood levels of the drugs in the patients were note measured, it cannot be ruled out that these two cases had lower blood levels of the drugs. The SP dose was developed for adults but in the main target group (children aged 2-5 years) the weight-adjusted dose produced blood concentrations of both components that are approximately half of those in adults [20]. This means that the standard dose may be sub-optimal in younger children. Although in the present study AS + SP shows high efficacy resulting in a high cure rate, there is still a need for close monitoring of the therapeutic efficacy of this ACT in Yemen. Resistance to the SP component drugs are easy to induce experimentally [21]. In Africa SP resistance developed soon after the drug was adopted as first-line treatment against falciparum malaria [22]. This has been attributed mainly to the long elimination half-life of the SP components, which make it much easier for resistance to develop to this drug compared to the more rapidly eliminated antimalarials [23]. The pattern of use of anti-malarial drugs is believed to be a major factor in the emergence and spread of anti-malarial drug resistance [24]. Nevertheless, it is notable that despite high rates of self-medication, incomplete treatments and sub-standard drugs, the efficacy of $\mathrm{AS}+\mathrm{SP}$ remains high in Yemen, as shown in the present results $[11,25,26]$.

The findings of high efficacy of AS + SP against falciparum malaria are supported by the fact that at present there is no evidence that SP has lost its efficacy against falciparum malaria in Yemen. In 2005, in vivo and in vitro tests were conducted by Al-Kabsi et al. to determine the SP efficacy against P. falciparum isolates from 100 malaria patients in Tihamah, Yemen [27]. In the in vivo test, no clinical or parasitological failure occurred. The in vitro test results suggested that SP is still effective against $P$. falciparum in the study area.

Published studies with SP resistance-associated molecular markers in Yemen are few and have not provided consistent results about the prevalence of these markers. A molecular marker study in Meseimeer found a $5 \%$ prevalence of $d h f r$ Arg-59 mutation in 99 amplified samples, while the dhps Glu-540 was not detected in 119 
amplified samples [6, 7]. This was interpreted to suggest that the selection process had not reached dhps [6]. In a more recent work [28], four drug-resistant genes ( $p f c r t$, pfmdr1, dhfr, and dhps) were genotyped in 108 P. falciparum isolates collected in three sites in Yemen: Dhamar, Hodeidah and Taiz. The investigators concluded that the absence of the triple mutant $d h f r$ genotype (IRN) and dhps mutations supports the use of AS + SP as first-line therapy. They suggested that the previous report on the presence of C59 mutation could possibly be explained by inclusion of expatriates in the sample of patients studied. In another study, isolates from 90 patients with microscopically confirmed $P$. falciparum infection from Al-Hodaida were analysed for the molecular $d h f r 108 \mathrm{~N}$ by Abdul-Ghani et al. [29]. The mutation was detected among about $61 \%$ of $P$. falciparum isolates in its pure and mixed-type forms. They suggested that the high frequency of $d h f r 108 \mathrm{~N}$ among parasite isolates should be cause for concern about the efficacy of SP as partner with AS. However, dhfr 108 mutation is a first step and is followed by other mutations before significant resistance occurs [30]. The high prevalence of SP resistanceassociated genotypes reported from Sudan [31] and other countries in the region, such as Ethiopia [32] and Somalia [33], calls for close monitoring for the emergence of these genotypes in Yemen.

In the present study, the 28-day, PCR-uncorrected cure rate after treatment with AL is $100 \%$ in Bajil (95\% CI 95.1-100 \%), in Jabal-Al-Sharq it is 97.8 \% (95 \% CI 92.2$99.7 \%$ ) and in Tur Bani Qa'is it is 94.3 \% (95 \% CI 87.2$98.1 \%)$. The PCR-corrected, 28-day cure rate after AL in each of the three sites is $100 \%$. This is higher than the findings of a recently pooled analysis of a 28 -day, PCRcorrected parasitological cure rate of $97.1 \%$ in adults and $97.3 \%$ in children [34]. The two cases classified as LPF in Jabal-Al-Sharq study in the uncorrected analysis (PCR was not done) could be explained by low bio-availability of the drug but drug blood levels have not been done to confirm.

The effect of AL in the present study on microscopic gametocytaemia is consistent with findings from other endemic areas, which show a significant impact of $A L$ on gametocytaemia [35]. The data also demonstrate the superior efficacy of AL over AS + SP in clearance of microscopic gametocytaemia. This could be a factor in choosing between different ACT in the future, particularly in an area of low transmission, on the path to malaria elimination.

\section{Conclusions}

AS + SP remains a safe and effective first-line drug for the treatment of uncomplicated falciparum malaria in Yemen. However, monitoring the efficacy of this ACT should be continued since there is a high risk of failure of the SP component due to sub-therapeutic levels resulting from inadequate use of anti-malarial drugs in the country. Surveillance for SP resistance-associated molecular markers should also be monitored as a supportive tool to in vivo efficacy. AL is highly efficacious in Yemen and remains the appropriate option as a second-line treatment for uncomplicated falciparum malaria. AL shows higher efficacy than $\mathrm{AS}+\mathrm{SP}$ in the clearance of microscopic gametocytaemia.

\section{Abbreviations}

TET: therapeutic efficacy test; AL: artemether-lumefantrine; SP: sulfadoxinepyrimethamine; ACT: artemisinin-based combination therapy; AS+SP: artesunate+sulfadoxine-pyrimethamine; AS: artesunate; S: sulfadoxine; P: pyrimethamine; ETF: early treatment failure; LCF: late clinical failure; LPF: late parasitological failure; ACPR: adequate clinical and parasitological response; LFU: lost to follow-up; WTH: withdrawal; CQ: chloroquine; CRF: case record form; WBC: white blood cell; PCR: polymerase chain reaction.

\section{Authors' contributions}

AAA: analysis of data, literature review, writing the manuscript; NAS: overall technical supervision of the studies and entering data and writing report; AA: supervision of logistics and coordination; AM: field work Hajaa Governorate in Haja and Tur Bani Qa'is; MG: field work Ibb Governorate in Al Udayn; AY: field work Dhamar Governorate in Jabal Ash Sharq; MM: Field work Emran Governorate in Alqaflah; MA: Haja Governorate in shares; YA: field work, Al Hudeida Governorate in Bajil; HA and GZ: planning, coordination, manuscript review; MW: development of the study protocol, data cleaning and validation, manscript preparation; $\mathrm{AB}$ : data analysis and manuscript preparation; HM and RAN: molecular analysis and PCR classification of cases. All authors read and approved the final manuscript.

\section{Author details}

${ }^{1}$ College of Medicine, King Saud University, Riyadh, Saudi Arabia. ${ }^{2}$ The National Malaria Control Programme, Ministry of Public Health and Population, Sanaa, Yemen. ${ }^{3}$ Ministry of Public Health and Population, Sanaa, Yemen. ${ }^{4}$ Malaria Control and Elimination, Division of Communicable Diseases Control, World Health Organization Regional Office for the Eastern Mediterranean, Cairo, Egypt. ${ }^{5}$ Global Malaria Programme, World Health Organization, Geneva, Switzerland. ${ }^{6}$ Naval Medical Research Unit-3, Cairo, Egypt.

\section{Acknowledgements}

We would like to express our appreciation to all patients and parents and guardians of the children who participated in the study. We thank the health authorities in the Governorates for their collaboration. We appreciate the work of all staff of the sentinel sites.We are grateful to Dr Pascal Ringwald for his collaboration and advice. Financial support for the studies were provided by WHO through the Bill and Melinda Gates Foundation and by The Global Fund to Fight AIDS, Tuberculosis and Malaria.

\section{Competing interests}

The authors declare that they have no competing interests.

\section{Disclaimer}

$H A, A B, M W$ and $G Z$ are staff members of $W H O$. The authors alone are responsible for the views expressed in this publication and they do not necessarily represent the decisions, policy or views of WHO.

Received: 10 September 2015 Accepted: 27 October 2015

Published online: 14 November 2015

\footnotetext{
References

1. WHO. World malaria report, 2014. Geneva: World Health Organization; 2014.
} 
2. Snow RW, Amratia P, Zamani G, Mundia CW, Noor AM, Memish ZA, et al. The malaria transition on the Arabian Peninsula: progress toward a malaria-free region between 1960-2010. Adv Parasitol. 2013;82:205-51.

3. Bamaga A, Mahdy MA, Mahmud R, Lim YA. Malaria in Hadhramout, a southeast province of Yemen: prevalence, risk factors, knowledge, attitude and practices (KAPs). Parasit Vectors. 2014;7:351.

4. WHO. Report on the inter-country workshop on monitoring therapeutic efficacy of antimalarial drugs, Annex 5: main results of monitoring drug resistance of falciparum malaria by country. Sana'a, Republic of Yemen, 21-25 April 2002, WHO-EM/MAL/281/E/L. http://whqlibdoc.who.int/ emro/2002/WHO-EM_MAL_281_E_L.pdf. Accessed 11 June 2015.

5. WHO. Monitoring antimalarial drug resistance. Report of WHO Consultation, Geneva, Switzerland, 3-5 December 2001. WHO monographs WHO/ CDS/CSR/EPH/2002.7.

6. Abdul-Kader R. Studies on drug resistant Plasmodium falciparum malaria in Yemen, PhD Thesis, University of Liverpool, 2006. http://library.liv. ac.uk/search S8?/ITHESIS+20651.MUB/Ithesis+20651+mub/-3\%2C$\% 2$ Co\%2CE/frameset\&FF =|thesis $+20651+$ mub\&1\%2C1\%2C. Accessed 24 Feb 2011.

7. Mubjer RA, Adeel AA, Chance ML, Hassan AA. Molecular markers of antimalarial drug resistance in Lahj Governorate, Yemen: baseline data and implications. Malar J. 2011;10:245.

8. WHO. Susceptibility of Plasmodium falciparum to antimalarial drugs: report on global monitoring: 1996-2004. WHO/HTM/MAL/2005.

9. Bloland P. Drug resistance in malaria. World Health Organization, Geneva, WHO/CDS/CSR/DRS/2001.4

10. Al-Maktari MT, Bassiouny HK. Malaria status in Al-Hodeidah Governorate, Republic lof Yemen. Part Il: human factors causing the persistence of chloroquine resistant $P$. falciparum local strain. J Egypt Soc Parasitol. 2003;33:829-39.

11. Bin Ghouth AS. Availability and prescription practice of anti-malaria drugs in the private health sector in Yemen. J Infect Dev Ctries. 2013;7:404-12.

12. WHO. Guidelines for the Treatment of Malaria, 3rd edn. World Health Organization: Geneva. 2015. http://www.who.int/malaria/publications/ atoz/9789241549127/en/.

13. Adjuik M, Babiker A, Garner P, Olliaro P, Taylor W, White N, et al. Artesunate combinations for treatment of malaria: meta-analysis. Lancet. 2004;363:9-17.

14. von Seidlein L, Milligan P, Pinder M, Bojang K, Anyalebechi C, Gosling $R$, et al. Efficacy of artesunate plus pyrimethamine-sulphadoxine for uncomplicated malaria in Gambian children: a double-blind, randomised, controlled trial. Lancet. 2000;355:352-7.

15. Omari A, Gamble C, Garner P. Artemether-lumefantrine for treating uncomplicated falciparum malaria. Cochrane Database Syst Rev. 2007;18:CD003125.

16. WHO. Methods for Surveillance of antimalarial drug efficacy. World Health Organization: Geneva. 2009. http://www.who.int/malaria/publications/ atoz/9789241597531/en/. Accessed 23 Oct 2015.

17. WHO. Methods and techniques for clinical trials on anti-malarial drug efficacy: genotyping to identify parasite populations. World Health Organization: Geneva. 2008. http://www.who.int/malaria/publications/ atoz/9789241596305/en/. Accessed 23 Oct 2015.

18. Dondorp AM, Nosten F, Yi P, Das D, Phyo AP, Tarning J, et al. Artemisinin resistance in Plasmodium falciparum malaria. N Engl J Med. 2009:361:455-67.

19. Noedl H, Se Y, Schaecher K, Smith BL, Socheat D, Fukuda MM. Evidence of artemisinin-resistant malaria in western Cambodia. N Engl J Med. 2008;359:2619-20.

20. Barnes KI, Little F, Smith PJ, Evans A, Watkins WM, White NJ. Sulfadoxinepyrimethamine pharmacokinetics in malaria: pediatric dosing implications. Clin Pharmacol Ther. 2006;80:582-96.

21. Gregson A, Plowe C. Mechanisms of resistance of malaria parasites to antifolates. Pharmacol Rev. 2005;57:117-45.
22. Bredenkamp B, Sharp B, Mthembu SD, Durrheim DN, Barnes KI. Failure of sulfadoxine-pyrimethamine in treating Plasmodium falciparum malaria in KwaZulu-Natal. S Afr Med J. 2001;91:970-1.

23. Watkins WM, Mosobo M. Treatment of Plasmodium falciparum malaria with pyrimethamine-sulfadoxine: selective pressure for resistance is a function of long elimination half- life. Trans R Soc Trop Med Hyg. 1993;87:75-8

24. White N. Antimalarial drug resistance and combination chemotherapy. Philos Trans R Soc Lond Biol Sci. 1999;354:739-49.

25. Abdo-Rabbo A, Bassili A, Atta H. The quality of antimalarials available in Yemen. Malar J. 2005;4:28.

26. Abdul-Ghani R, Farag HF, Allam AF, Shawky SM, Al-Mekhlafi AM. Mutant Plasmodium falciparum chloroquine resistance transporter in Hodeidah, Yemen : association with parasitologic indices and treatment-seeking behaviors. Acta Trop. 2013;128:473-8.

27. Al-Kabsi AM, Al-Shamahy HA, Al-Harazy AH, Harmal NS. The therapeutic efficacy of sulfadoxine/pyrimethamine against Plasmodium falciparum in Yemen. Med Princ Pract. 2009;18:62-6.

28. Al-Hamidhi S, Mahdy MA, Al-Hashami Z, Al-Farsi H, Al-Mekhlafi AM, Idris MA, et al. Genetic diversity of Plasmodium falciparum and distribution of drug resistance haplotypes in Yemen. Malar J. 2013;12:244.

29. Abdul-Ghani R, Farag HF, Allam AF, Shawky SM. Prevailing Plasmodium falciparum dihydrofolate reductase 108-asparagine in Hodeidah, Yemen: a questionable sulfadoxine-pyrimethamine partner within the artemisinin-based combination therapy. Acta Trop. 2014;132:39-44.

30. Lozovsky ER, Chookajorn T, Brown KM, Imwong M, Shaw PJ, Kamchonwongpaisan S, et al. Stepwise acquisition of pyrimethamine resistance in the malaria parasite. Proc Natl Acad Sci USA. 2009;106:12025-30.

31. Gadalla NB, Abdallah TM, Atwal S, Sutherland CJ, Adam I. Selection of pfdhfr/pfdhps alleles and declining artesunate/sulphadoxine-pyrimethamine efficacy against Plasmodium falciparum 8 years after deployment in eastern Sudan. Malar J. 2013;12:255.

32. Mula P, Fernández-Martínez A, de Lucio A, Ramos JM, Reyes F, González V, et al. Detection of high levels of mutations involved in anti-malarial drug resistance in Plasmodium falciparum and Plasmodium vivax at a rural hospital in southern Ethiopia. Malar J. 2011;10:214.

33. Warsame M, Hassan AM, Barrette A, Jibril AM, Elmi HH, Arale AM, et al. Treatment of uncomplicated malaria with artesunate plus sulfadoxinepyrimethamine is failing in Somalia: evidence from therapeutic efficacy studies and Pfdhfr and Pfdhps mutant alleles. Trop Med Int Health. 2015;20:510-7.

34. Makanga M, Bassat Q, Falade CO, Premji ZG, Krudsood S, Hunt P, et al. Efficacy and safety of artemether-lumefantrine in the treatment of acute, uncomplicated Plasmodium falciparum malaria: a pooled analysis. Am J Trop Med Hyg. 2011;85:793-804.

35. Makanga MA. Review of the effects of artemether-lumefantrine on gametocyte carriage and disease transmission. Malar J. 2014;13:291.

\section{Submit your next manuscript to BioMed Central and take full advantage of:}

- Convenient online submission

- Thorough peer review

- No space constraints or color figure charges

- Immediate publication on acceptance

- Inclusion in PubMed, CAS, Scopus and Google Scholar

- Research which is freely available for redistribution

Submit your manuscript at

www.biomedcentral.com/submit

C BioMed Central 